1 Universidade Federal de Santa Catarina (UFSC), Programa de Pós-Graduação em Saúde Coletiva - Florianópolis (SC), Brasil.

luisanlosso@gmail.com

2 Universidade Federal de Santa Catarina (UFSC), Programa de Pós-Graduação em Saúde Coletiva - Florianópolis (SC), Brasil sergio.freitas@ufsc.br

\section{Avaliação do grau da implantação das práticas integrativas e complementares na Atenção Básica em Santa Catarina, Brasil}

\author{
Implementation level assessment of integrative and complementary \\ practices in Primary Health Care in Santa Catarina, Brazil
}

Luisa Nuernberg Losso ${ }^{\mathbf{1}}$, Sérgio Fernando Torres de Freitas ${ }^{\mathbf{2}}$

RESUMO Esta pesquisa desenvolveu e aplicou um modelo de avaliação do grau de implantação das Práticas Integrativas e Complementares (PIC) na Atenção Básica nos municípios de Santa Catarina (SC) utilizando dados do segundo ciclo da avaliação externa do Programa Nacional de Melhoria do Acesso e da Qualidade da Atenção Básica (PMAQ-AB). O modelo demonstrou viabilidade de aplicação nos 293 municípios de SC, classificando-os por graus de implantação: 35 municípios (11,9\%) apresentaram implantação incipiente; 46 (15,7\%) estavam parcialmente implantados; 50 (17,1\%), implantados; e 162 (55,3\%) municípios não ofertavam PIC. Este modelo serve para avaliar as PIC em outros estados do Brasil.

PALAVRAS-CHAVE Avaliação em saúde. Terapias complementares. Medicina integrativa. Atenção Primária à Saúde. Qualidade, acesso e avaliação da assistência à saúde.

ABSTRACT This research developed and applied and assessment model of implementation levels as for the Integrative and Complementary Practices (PIC) in Primary Care in the municipalities of the State of Santa Catarina by means of data provided by the second phase of National Program for Access and Quality Improvement in Primary Care - PMAQ-AB's database. The empirical application of the model demonstrated to be feasible in the 293 municipalities of Santa Catarina since it was able to classify them as to levels of implementation: 35 municipalities (11.9\%) revealed incipient level of implementation; 46 (15.7\%) had partially implemented; 50 (17.1\%) had fully implemented; and 162 (55.3\%) municipalities did not deploy the service. The model here designed is adequate to evaluate the implementation of the PIC in other Brazilian states.

KEYWORDS Health evaluation. Complementary therapies. Integrative medicine. Primary Health Care. Health care quality, access and evaluation. 


\section{Introdução}

As Práticas Integrativas e Complementares (PIC) fazem parte de um campo de cuidados em saúde que engloba as racionalidades médicas vitalistas e práticas terapêuticas ditas integrativas e complementares em saúde, também definidas pela Organização Mundial da Saúde (OMS) como Medicina Tradicional, Complementar e Alternativa (NASCIMENTO ET AL., 2013; WHO, 2013).

No Brasil, a Política Nacional de Práticas Integrativas e Complementares (PNPIC) faz parte de uma estratégia do Ministério da Saúde (MS) para implantação de ações e serviços relativos às $\mathrm{PIC}$ no Sistema Único de Saúde (SUS) em todo território nacional (BRASIL, 2006A).

As práticas descritas na PNPIC são representadas pelas racionalidades médicas das medicinas tradicional chinesa, homeopática e antroposófica, pelas práticas terapêuticas da fitoterapia, plantas medicinais, e pelo termalismo social ou a crenoterapia. Elas têm por objetivo estimular mecanismos naturais de prevenção de agravos e recuperação da saúde, ampliando a oferta e as opções terapêuticas para um novo modelo de atenção à saúde e cuidado aos usuários do SUS (BRASIL, 2012C).

As diretrizes e ações da PNPIC estão em convergência com políticas nacionais, como a da Atenção Básica (AB), de promoção da saúde, de humanização, de educação popular, de educação permanente, de povos e comunidades tradicionais, dentre outras, cujas interações são recomendadas pelo MS como estratégia de ampliação e integração das PIC em diferentes contextos e comunidades, permitindo mais opções terapêuticas aos usuários do SUS (BRASIL, 2012C).

Com a publicação da PNPIC, o Departamento de Atenção Básica (DAB) foi eleito o órgão responsável pela gestão nacional das PIC, desempenhando o papel de normatização, monitoramento, sensibilização e divulgação (CAVALCANTIET AL., 2014).

A PNPIC incentiva a implantação das PIC no âmbito do SUS, mas não define quais ações e recursos são necessários para tal, dificultando sua consolidação e tornando sua implantação, frente a tais condições, um desafio aos gestores públicos em saúde (CAVALCANTI ET AL., 2014).

Muitos levantamentos têm sido realizados pelo MS e DAB sobre a oferta dos serviços de PIC e sua expansão pelo território brasileiro, mas ainda não foram divulgados indicadores de avaliação desse serviço no SUS, reconhecido pela PNPIC como uma ação de responsabilidade federal (BRASIL, 2006A).

Com o intuito de incentivar a melhoria dos serviços de $\mathrm{AB}$ em todo o território nacional, em 2011, foi criado pelo MS o Programa Nacional de Melhoria do Acesso e da Qualidade da Atenção Básica (PMAQ$\mathrm{AB})$, que incentiva os gestores a melhorar a qualidade dos serviços de saúde ofertados à população. Via um conjunto de estratégias de qualificação, acompanhamento e avaliação por meio da avaliação das Equipes de Atenção Básica (EAB) e oferecimento de gratificações pelo desempenho individual de cada equipe as PIC são avaliadas pelo PMAQ-AB desde o primeiro ciclo de coleta como um aspecto de prioridade da Política Nacional de Atenção Básica (PNAB), reconhecendo o esforço de equipes que tenham empreendido iniciativas que estimulem a ampliação do acesso nos diversos contextos existentes no Brasil (BRASIL, 2011, 2012B, 2013A).

Considerando a existência desse serviço no SUS, a necessidade de avaliar sua implantação e a existência de dados de PIC através do PMAQ-AB, a pesquisa aqui relatada teve como objetivo avaliar o grau de implantação das PIC na AB em Santa Catarina utilizando os dados provenientes da avaliação externa PMAQ-AB no ciclo de 2013.

\section{Metodologia}

Esta é uma pesquisa avaliativa do grau de implantação das PIC na AB nos municípios catarinenses e se baseia na análise de normativas 
do MS e DAB por meio de dados secundários. O critério utilizado nesta avaliação é o da eficácia, dado que objetiva identificar se as recomendações para implantação propostas estão sendo implementadas conforme preconizado pelas instâncias superiores (MS e DAB), de forma a alcançar as metas propostas. O critério de eficácia adotado é aquele proposto por Silva e Formigli (1994), está relacionado com o efeito das ações e práticas de saúde implementadas, e visa a compreender o cumprimento de objetivos ou metas.

Os dados são referentes ao banco da avaliação externa do PMAQ-AB em 2013 em Santa Catarina, cuja coleta foi realizada em parceria do MS com instituições de ensino e pesquisa do Brasil. A população residente de Santa Catarina no ano de 2013 foi coletada no site do Departamento de Informática do Sistema Único de Saúde (Datasus), sendo a fonte dos dados o Instituto Brasileiro de Geografia e Estatística (IBGE).

A amostra contemplou 1470 equipes, que atendem a 293 municípios de Santa Catarina. Embora o PMAQ tenha coletado dados por adesão voluntária, é importante destacar que Santa Catarina foi o estado que mais aderiu, posto que havia $91,2 \%$ das EABs localizadas na totalidade dos municípios do Estado (BRASIL, 2013B).

O estado de Santa Catarina está localizado na região sul do Brasil e sua estimativa populacional foi de 6.634.254 habitantes para o ano de 2013, conforme o IBGE, distribuídos de forma heterogênea nos 295 municípios, alocados em nove macrorregiões de saúde. Possui 229 municípios com até 20 mil habitantes, sendo caracterizado pela prevalência de pequenos municípios (78\%) (BRASIL, 2013A).

Os dados do DAB consideraram a existência de 293 municípios no ano de 2013, porque dois municípios foram criados no final desse ano e não apresentavam estrutura própria de saúde instalada no momento da coleta de dados com as equipes.

As informações selecionadas foram retiradas de dois módulos do PMAQ-AB, na área específica de PIC. O Módulo I contém informações relativas às Unidades Básicas de Saúde e seus insumos e materiais para PIC; e o Módulo II, correspondente à $\mathrm{EAB}$, apresenta informações sobre oferta e descrição do serviço de PIC, ações das equipes em educação popular com a comunidade e desenvolvimento de ações de educação permanente pela gestão.

Realizou-se análise de consistência dos dados, o que permitiu a identificação de erros ou duplicação de casos, reconhecidos pela presença de mais de dois registros com a mesma informação nas equipes, sendo realizadas as devidas correções.

O banco de dados formado pelas variáveis do PMAQ-AB e dos sistemas de informações (Datasus) foram apresentados e analisados no programa Microsoft Office Excel 2007 ${ }^{\circledR}$.

Elaborou-se modelo avaliativo que permitisse avaliar o grau de implantação das PIC na AB. A construção desse modelo foi realizada a partir de levantamento do referencial teórico; da fundamentação nas normativas; da oficina de consenso; e do instrumento de avaliação externa no PMAQ-AB, o que orientou a elaboração do modelo teórico-lógico do grau de implantação das PIC na AB apresentado na figura 1. 
Figura 1. Modelo teórico-lógico do grau de implantação das PIC na Atenção Básica.

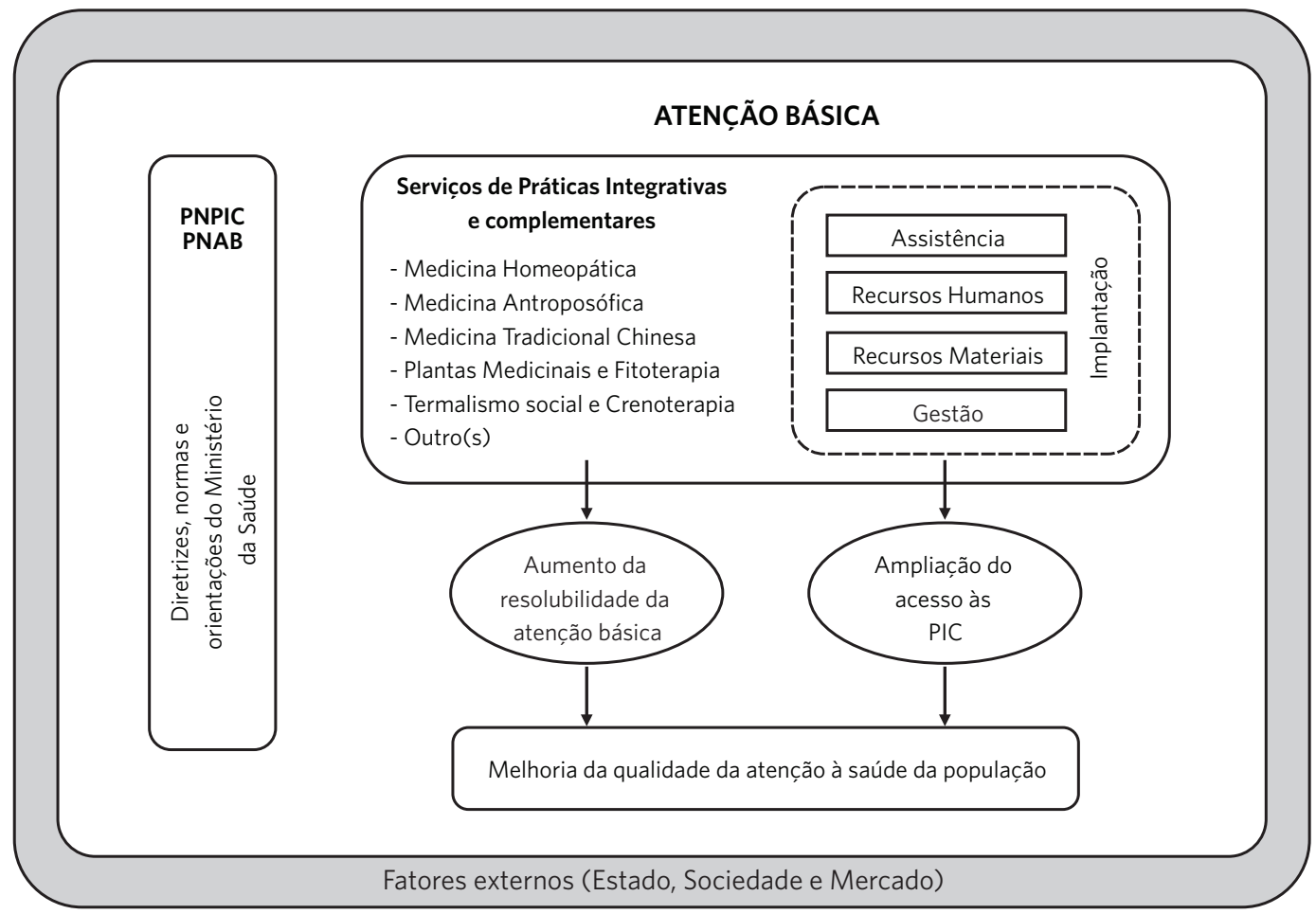

Fonte: Elaboração própria.

Segundo Hartz e outros (1997), para a construção de um modelo teórico-lógico existem fatores importantes a serem analisados, incluindo dimensões macro e microexplicativas. Estas últimas compõem a micro e macroteoria de um objeto, sendo que a primeira se relaciona a normas, aspectos estruturais e operacionais; e a outra detalha o contexto de implantação do objeto, por meio dos fatores organizacionais e sociopolíticos que favorecem ou inibem o sucesso da implantação.

Nesse sentido, a figura 1 está apresentada sob o contexto de determinantes sociais, econômicos e políticos que influenciam a existência do modelo teórico-lógico, implicado por fatores externos que se traduzem pela variedade de interesses e a permanente negociação entre as lógicas do Estado que normatiza e regula, da Sociedade que financia, e do Mercado que produz e oferta os serviços. Essas relações de negociação permanente entre os diferentes interesses influenciam a oferta de serviços de saúde mesmo que no âmbito exclusivo da esfera pública, por exemplo, na regulação de insumos e dos recursos humanos. Reconhece-se também que o modelo está inserido em um ambiente de políticas públicas, onde se disputam diferentes interesses, modelos de atenção e de cuidado (BAHIA, 2009; BRASIL, 2012A; CONTANDRIOPOULOS, 1996; MEDINA ET AL., 2005). Ressalta-se que o modelo construído é orientado para a gestão, visto que o gestor é quem possui maior governabilidade para o processo de implantação, organização, estruturação e funcionamento dos serviços no setor saúde.

Os principais documentos a fundamentar a consolidação do modelo teórico-lógico foram a Portaria GM no ${ }^{\circ} 488 / 11$ - PNAB (BRASIL, 2012B); a Portaria GM no 971/2006 - PNPIC (BRASIL, 2006A); e a Portaria SAS no 853/2006 - que cria o serviço de PIC no SUS e o inclui na Tabela 
de Serviços e classificações do Sistema de Cadastro Nacional de Estabelecimentos de Saúde (SCNES) (BRASIL, 2006B). O modelo apresenta o serviço de PIC, definido pela PNPIC por três sistemas médicos complexos de racionalidades vitalistas, quis sejam, a Medicina Tradicional Chinesa (MTC), a medicina homeopática e a medicina antroposófica, e duas práticas terapêuticas ditas complementares e integrativas em saúde, que são a fitoterapia e plantas medicinais, além do termalismo social ou a crenoterapia.

Considerou-se que, para sua implantação na $A B$, o serviço das PIC deve ser avaliado em quatro dimensões: Assistência, Recursos Humanos, Recursos Materiais e Gestão. A dimensão 'assistência' corresponde às ações de atenção à saúde na área de PIC e faz referência à oferta e distribuição do serviço de PIC no município; a dimensão Recursos Humanos contempla o desenvolvimento de ações de educação popular em saúde com as PIC, tendo em vista ações das equipes com a comunidade; a dimensão Recursos Materiais dispõe sobre os materiais e insumos para o atendimento clínico na área e tem a finalidade de avaliar a disponibilidade desses materiais nos municípios; e a dimensão Gestão corresponde à responsabilidade da gestão municipal em investir na capacitação dos profissionais na área de PIC, ofertando educação continuada na forma de educação permanente com vistas ao aprimoramento para o trabalho.

A seleção de indicadores fez-se por meio das normativas e do instrumento de avaliação externa no PMAQ-AB e foi validada em oficina de consenso com os especialistas, na forma de comitê tradicional, onde foram contraídos pactos entre especialistas das áreas de avaliação em saúde e PIC, tendo por objetivo validar os indicadores e medidas e gerar maior legitimidade ao modelo avaliativo elaborado (MEDINA ET AL., 2005).

Entregou-se previamente aos participantes o material composto pelo modelo teórico-lógico e matriz avaliativa contendo o julgamento dos indicadores, dimensões e implantação. Ao final de cada instrumento, constava um quadro para preenchimento de escala de concordância e observações para o juízo de valor de cada participante. Ainda acompanhava informações, como o dicionário das variáveis e o rationale das dimensões.

O modelo teórico-lógico foi discutido durante a oficina e não sofreu alterações substanciais, mas foi solicitado o detalhamento de alguns elementos, como a apresentação na ilustração das principais políticas orientadoras do processo de implantação das PIC, e a descrição dos resultados com a implantação do serviço de PIC, dando ênfase ao aumento da resolubilidade na $\mathrm{AB}$ e da ampliação do acesso às PIC.

A oficina prosseguiu com a discussão da matriz avaliativa, o que resultou em sua alteração. As dimensões foram mantidas, o número de indicadores foi diminuído de dez para seis, houve adição de medidas e alteração no peso do julgamento das dimensões. Nesses casos, tais contribuições foram incorporadas ao modelo final.

Os dados utilizados no estudo são de domínio público, não tendo sido necessária autorização do Comitê de Ética em Pesquisa.

\section{Desenvolvimento da matriz de avaliação e julgamento}

O modelo final da matriz avaliativa definido na oficina de consenso comporta quatro dimensões, seis indicadores, oito medidas, bem como seus respectivos parâmetros de julgamento para cada indicador e dimensão (quadro 1), os quais permitiram formular um modelo de classificação para juízo do grau de implantação das práticas integrativas e complementares na $\mathrm{AB}$ (figura 2). O consenso entre os especialistas foi necessário para definir parâmetros e medidas, visto que não há normativa que estabeleça valores padrão, considerando-o um modelo empírico. 
Quadro 1. Matriz avaliativa, com descrição das variáveis, medidas e parâmetros para a classificação dos municípios quanto ao grau de implantação das PIC na Atenção Básica

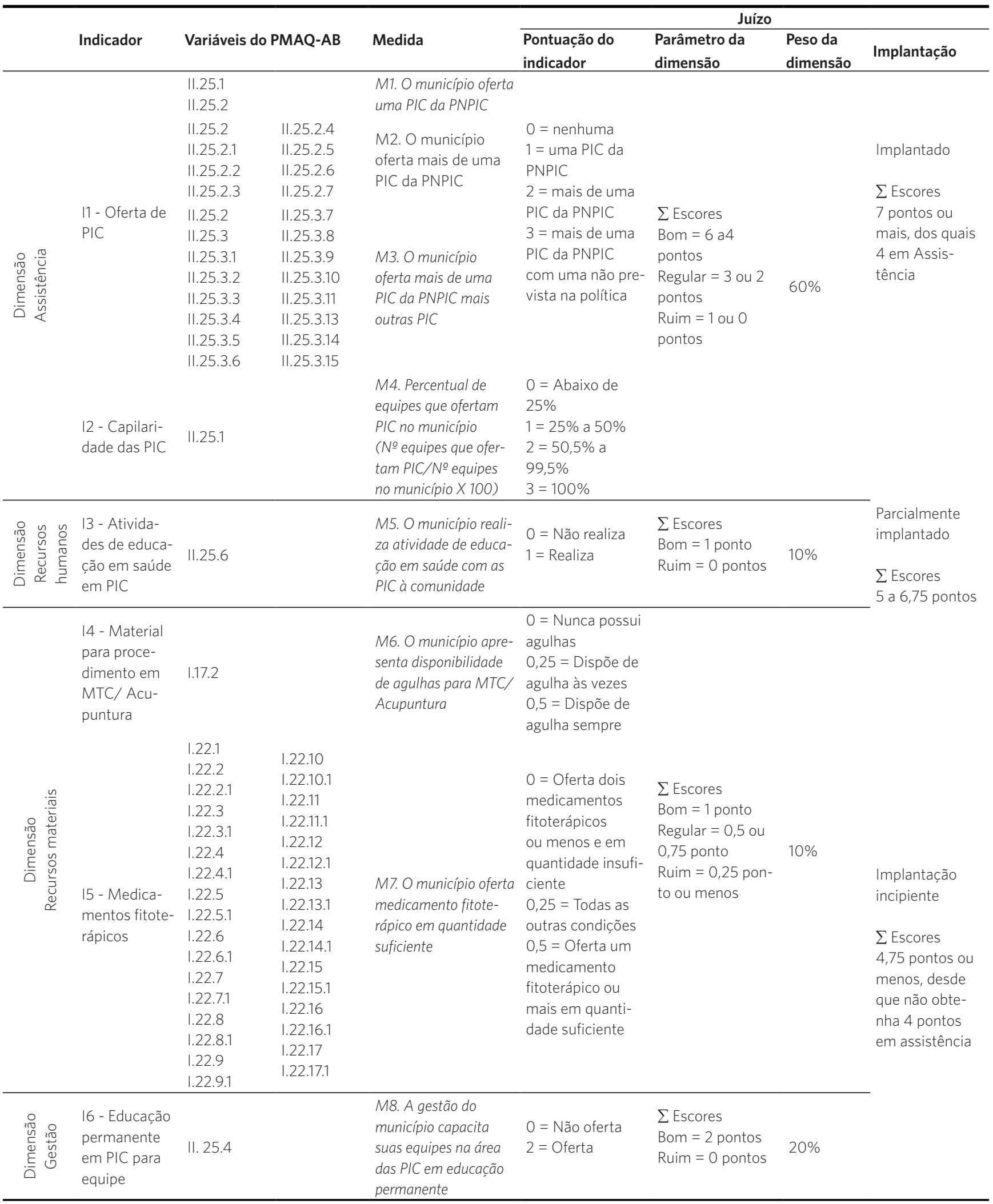

Fonte: Elaboração própria. 
No quadro 1, observam-se os componentes das dimensões Assistência, Recursos Humanos, Recursos Materiais e Gestão. A dimensão Assistência refere-se à oferta e distribuição do serviço de práticas integrativas e complementares nos municípios. Essa dimensão apresenta dois indicadores: (1) Oferta de PIC: avalia a existência do serviço de PIC nos municípios por meio das PIC registradas na Portaria GM/ MS $n^{0} 853 / 2006$, que permite o registro de sete classificações estabelecidas na PNPIC e avalia a existência de outras PIC não contempladas na Portaria no 971/2006 - PNPIC; (2) Capilaridade das PIC: pressupõe o percentual ideal de equipes que ofertam PIC em cada município, identificando, assim, a capilaridade e abrangência dos serviços pelo município.

A dimensão Recursos Humanos (quadro 1) propõe a análise das EABs e seu desenvolvimento como equipes capacitadas e ampliadas para realizar diferentes ações na área de PIC em cada município, apresentando o indicador Atividades de educação em saúde em PIC, que avalia a realização dessas atividades na área de PIC desenvolvidas pelas equipes com a comunidade, no município.

A dimensão Recursos Materiais (quadro 1) dispõe sobre os materiais e insumos necessários para atendimento e procedimentos com as PIC, contemplando dois indicadores. Esses indicadores definem o mínimo necessário em termos de recursos materiais para que se exerçam duas das PIC mais comumente ofertadas no SUS: (1) Material para procedimento em Medicina Tradicional Chinesa - Acupuntura (MTC-acupuntura): verifica a disponibilidade de materiais para a realização de acupuntura, considerando que o material essencial e indispensável são as agulhas filiformes descartáveis (BRASIL, 2006B); (2) Medicamentos fitoterápicos: avalia a disponibilidade do medicamento fitoterápico em quantidade suficiente no município, como fixado na Relação Nacional de Medicamentos Essenciais (Rename) (BRASIL, 2012A).

A dimensão Gestão (quadro 1) compreende o compromisso do gestor municipal com a capacitação da equipe, apresentando o indicador Educação permanente em PIC para equipe, que avalia a oferta de educação permanente pelo gestor, identificando o compromisso com a capacitação profissional na área de práticas integrativas e complementares.

Quanto à etapa de agregação para obtenção dos valores dos municípios, as medidas foram calculadas a partir de duas possibilidades, que dependiam da existência de uma ou mais equipes em cada município: (1) Quando existe uma única equipe, o cálculo da medida do critério é igual à medida do indicador; (2) Quando há mais de uma equipe no município, a medida do critério é calculada a partir do maior valor da equipe, ou seja, a melhor experiência em PIC que apresentasse a melhor condição para o município. Concluindo, se pelo menos uma equipe do município apresentasse as condições mínimas estabelecidas como ideais a partir dos parâmetros do modelo avaliativo elaborado, o município seria bem avaliado, mesmo que suas outras equipes apresentassem menor pontuação. $\mathrm{O}$ único indicador que diferencia os municípios pela quantidade de equipes é o indicador de Capilaridade das PIC, que calcula o percentual de equipes que ofertam PIC no município.

A partir da matriz avaliativa, foi possível criar um julgamento para implantação das PIC na AB no ano de 2013, classificando os municípios em graus de implantação, conforme apresentado na figura 2. As dimensões apresentaram pesos diferentes, tendo a dimensão Assistência 60\%, as dimensões Recursos Humanos e Recursos Materiais, $10 \%$ cada uma e a dimensão Gestão, 20\%. A agregação dos escores permitiu classificar os municípios com o seguinte juízo de valor sobre sua implantação:

Implantado: Elevado grau de implantação; municípios que apresentem escore final de 7 ou mais, desde que obtenham escore 4 na dimensão Assistência;

Parcialmente implantado: Médio grau de 
implantação; municípios que apresentem escores de 5 a 6,75 ;

Implantação incipiente: Baixo grau de implantação; municípios que apresentem escores iguais ou menor que 4,75, desde que não obtenham escore 4 na dimensão Assistência. Neste caso serão realocados para o juízo de valor 'parcialmente implantado'.

Convém ressaltar que apenas as dimensões Assistência e Recursos Materiais foram avaliadas segundo os juízos de valor Ruim, Regular e Bom. As demais, Recursos Humanos e Gestão, foram avaliadas em Ruim ou Bom.

\section{Resultados e discussão}

Os resultados e a discussão apresentados a seguir estão organizados por dimensões e classificação final dos municípios catarinenses quanto ao grau de implantação das PIC na $\mathrm{AB}$ catarinense.

\section{Aplicabilidade do modelo de avaliação}

Das 1470 equipes avaliadas, 428 (29,1\%) equipes ofertavam alguma PIC. Dos $293 \mathrm{mu}$ nicípios, 131 (44,7\%) ofertavam alguma PIC contemplada na PNPIC e, dentre estes, 85 ofertavam outras PIC não contempladas na política. Das práticas previstas na PNPIC, são ofertadas pelos municípios: plantas medicinas ou fitoterapia (42,1\%); acupuntura $(17,7 \%)$; homeopatia $(16,3 \%)$; auriculoterapia $(12,4 \%)$; práticas corporais $(7,7 \%)$; termalismo social $(2,4 \%)$; e antroposofia (1,4\%). Dentre as práticas não previstas na PNPIC, estão a terapia comunitária (22,6\%); musicoterapia (11,3\%); e shantala $(9,9 \%)$. Há municípios que ofertam do in, shiatshu, massoterapia e reflexoterapia $(8,0 \%)$; arteterapia ( $8,0 \%)$; florais $(6,6 \%)$; yoga e dança circular em mesmo percentual $(6,1 \%)$; naturologia (4,7\%); biodança e geoterapia em mesmo percentual (3,8\%); ayurveda e talassoterapia em mesmo percentual (1,4\%); e sistema rio aberto $(0,9 \%)$.

O estudo de Sousa et al. (2012) demonstrou grande limitação na mensuração e avaliação das PIC associada aos sistemas de informação atuais, os quais não permitem o registro adequado dessas práticas. Um fator positivo da avaliação externa do PMAQ-AB é que ela permite identificar a oferta de outras práticas não contempladas na PNPIC.

Optou-se por desenvolver a análise das dimensões sobre do juízo de valor desta avaliação com base no porte populacional dos municípios de $\mathrm{SC}$, classificados em quatro estratos: até 5.000 habitantes (hab.); $5.001 \mathrm{a}$ 20.000 hab.; de 20.001 a 50.000 hab.; e mais de 50.000 hab. Esses estratos permitiram comparações tanto com a classificação do IBGE para municípios de pequeno, médio e grande porte como com outros estudos com dados do PMAQ-AB (tabela 1). 
Tabela 1. Distribuição de municípios segundo porte, dimensão de análise e juízo de valor na avaliação do grau de implantação das práticas integrativas e complementares na Atenção Básica em Santa Catarina, Brasil, 2013

\begin{tabular}{|c|c|c|c|c|c|c|}
\hline \multirow[t]{2}{*}{ Classificação } & \multicolumn{2}{|c|}{ Ruim } & \multicolumn{2}{|c|}{ Regular } & \multicolumn{2}{|c|}{ Bom } \\
\hline & $n$ & $\%$ & $n$ & $\%$ & $n$ & $\%$ \\
\hline \multicolumn{7}{|l|}{ Assistência } \\
\hline$\leq 5$ mil hab $(n=35)$ & 0 & 0,0 & 1 & 2,9 & 34 & 97,1 \\
\hline $5 f 20$ mil hab $(n=48)$ & 5 & 10,4 & 18 & 37,5 & 25 & 52,1 \\
\hline $20-50$ mil hab $(n=25)$ & 4 & 16,0 & 11 & 44,0 & 10 & 40,0 \\
\hline$>50$ mil hab $(n=23)$ & 4 & 17,4 & 10 & 43,5 & 9 & 39,1 \\
\hline Total & 14 & 10,7 & 40 & 30,5 & 77 & 58,8 \\
\hline \multicolumn{7}{|l|}{ Recursos Humanos ${ }^{\star}$} \\
\hline$\leq 5$ mil hab $(n=35)$ & 8 & 22,9 & - & - & 27 & 77,1 \\
\hline $5-20$ mil hab $(n=48)$ & 11 & 22,9 & - & - & 37 & 77,1 \\
\hline $20-150$ mil hab $(n=25)$ & 3 & 12,0 & - & - & 22 & 88,0 \\
\hline$>50$ mil hab $(n=23)$ & 3 & 13,0 & - & - & 20 & 87,0 \\
\hline Total & 26 & 19,9 & & & 105 & 80,1 \\
\hline \multicolumn{7}{|l|}{ Rercursos Materiais } \\
\hline$\leq 5$ mil hab $(n=35)$ & 9 & 25,7 & 22 & 62,9 & 4 & 11,4 \\
\hline 5 - 20 mil hab $(n=48)$ & 28 & 58,3 & 18 & 37,5 & 2 & 4,2 \\
\hline $20-50$ mil hab $(n=25)$ & 9 & 36,0 & 16 & 64,0 & 0 & 0,0 \\
\hline$>50$ mil hab $(n=23)$ & 11 & 47,8 & 8 & 34,8 & 4 & 17,4 \\
\hline Total & 57 & 43,5 & 64 & 48,9 & 10 & 7,6 \\
\hline \multicolumn{7}{|l|}{ Gestão* } \\
\hline$\leq 5$ mil hab $(n=35)$ & 17 & 48,6 & - & - & 18 & 51,4 \\
\hline $5-20$ mil hab $(n=48)$ & 21 & 43,8 & - & - & 27 & 56,3 \\
\hline $20-150$ mil hab $(n=25)$ & 12 & 48,0 & - & - & 13 & 52,0 \\
\hline$>50$ mil hab $(n=23)$ & 9 & 39,1 & - & - & 14 & 60,9 \\
\hline Total & 59 & 45,0 & & & 72 & 55,0 \\
\hline
\end{tabular}

Fonte: Elaboração própria.

*As dimensões Recursos Humanos e Gestão possuíam respostas dicotômicas.

A tabela 1 apresenta um perfil dos $131 \mathrm{mu}-$ nicípios catarinenses que foram avaliados e apresentam alguma PIC contemplada na PNPIC, permitindo a análise segundo o juízo de valor das dimensões.

Os dados demonstraram que, na dimensão Assistência, a maioria dos municípios apresentou a classificação 'bom' (58,8\%), com destaque para os municípios com menos de $5 \mathrm{mil}$ habitantes. De maneira geral, os municípios até cinco mil habitantes apresentaram bons indicadores, com a possibilidade de terem sido privilegiados na dimensão Assistência por possuir o peso maior no julgamento. Deve-se levar em consideração que o pactuado era que a avaliação valorizasse qualquer experiência em PIC e penalizasse os que não tivessem condições mínimas para ser considerados implantados. Quanto à análise do porte populacional na dimensão e o indicador de capilaridade das PIC, identificou-se que alguns municípios com mais de cinquenta mil 
habitantes que receberam pontuação baixa no indicador não apresentavam condições mínimas de cobertura populacional com as EABs que possuíam, refletindo no juízo de valor dado ao próprio indicador. Tais casos foram analisados separadamente, e a decisão foi manter os parâmetros de avaliação.

Em pesquisas de análise da oferta de serviços na $\mathrm{AB}$, uma das diferenças encontradas entre os municípios é a relação entre porte populacional e sua localidade. Municípios pequenos e localizados em áreas remotas demonstraram que a gestão municipal, em condições isoladas, tem dificuldade de responder pelo conjunto de ações estipulado pela PNAB (BRASIL, 2012B). Essa realidade é diferente daquela de municípios de grande porte, que possuem um apoiador institucional da gestão, o que facilita maior disponibilidade de recursos em nível organizacional, político-institucional e financeiro (FAUSTO ET AL., 2014; SEIDL ET AL., 2014).

Tais resultados convergem para a necessidade de políticas e ações voltadas para os municípios de pequeno porte, considerando que, se estes apresentassem condições favoráveis para a execução de ações na AB, contribuiriam para a consolidação de atendimento de qualidade e resolutivo para as EABs (SEIDL ET AL., 2014).

A dimensão Recursos Materiais foi considerada 'regular' e 'ruim' na maioria dos municípios, somando $92,4 \%$ das avaliações. Os indicadores de Recursos Materiais mereceram, em sua grande maioria, o juízo de valor 'regular', refletindo que o provimento dos medicamentos fitoterápicos e insumos estratégicos para MTC-Acupuntura possa apresentar deficiências na oferta regular, o que reflete na ação diária dos profissionais da $\mathrm{AB}$, dificultando a ampliação do acesso às PIC pelos usuários do SUS.

Outro estudo que investigou gestores e profissionais de saúde sobre a implantação das PIC no SUS, identificou dificuldades no fornecimento de material e aquisição de insumos utilizados em algumas das PIC, constituindo-se em grande problema na UBS (ISCHKANIAN; PELICIONI, 2012).
As dimensões Recursos Humanos (80,1\%) e Gestão (55\%) foram bem avaliadas. Estes resultados, embora sem associações diretas, sugerem que outros fatores sejam analisados, como é o caso da motivação dos profissionais. Constatou-se que, a partir do juízo de valor diferenciado entre eles, mesmo sendo dimensões que avaliam a capacitação profissional em PIC, a oferta de educação permanente pela gestão não pode ser associada somente à qualificação dos profissionais que realizam atividades de educação em saúde em PIC com a comunidade, já que pode também caracterizar o esforço singular dos profissionais pela sua auto capacitação. A avaliação destas dimensões também parece não ser influenciada pelo porte dos municípios, o que requer outras análises e pesquisas na área.

A literatura descreve que, no Brasil, embora haja um compartilhamento de responsabilidades entre os três entes da federação quanto à gestão e financiamento da $\mathrm{AB}$, cada município tem autonomia para realizar as ações de saúde em seu território, inclusive sobre as decisões relativas a recursos humanos, contratação, formação e capacitação, de acordo com a PNAB Nesse sentido, a ausência ou insuficiência de incentivos para a qualificação profissional pode contribuir para a desmotivação dos profissionais na AB (BRASIL, 2012B, SEIDL ET AL., 2014).

Pesquisas demonstram que é relevante para a gestão investir em iniciativas de educação permanente que fortaleçam a participação e o comprometimento dos profissionais, aliados aos interesses e necessidades demandados pelos trabalhadores de cada território. Esta afirmativa é consonante com os dados já apresentados, demonstrando a importância de se continuar empenhando esforços já existentes nas ações de educação permanente na área de PIC pelas equipes catarinenses (FONSECA SOBRINHO ET AL., 2014; SEIDL ET AL., 2014).

A oferta de educação permanente é uma ferramenta potente de gestão do trabalho, que valoriza o trabalhador e qualifica o seu processo de trabalho. Nesse sentido, destacam-se iniciativas de educação permanente, 
que associam as intervenções nos territórios e também as discussões entre suas equipes, com o aumento da chance de melhor certificação das EABs pelo PMAQ-AB (FONSECA
SOBRINHO ET AL., 2014; SEIDL ET AL. 2014).

\section{Classificação final dos municípios}

Figura 2. Mapa dos municípios avaliados segundo grau de implantação das PIC em Santa Catarina, Brasil, 2013

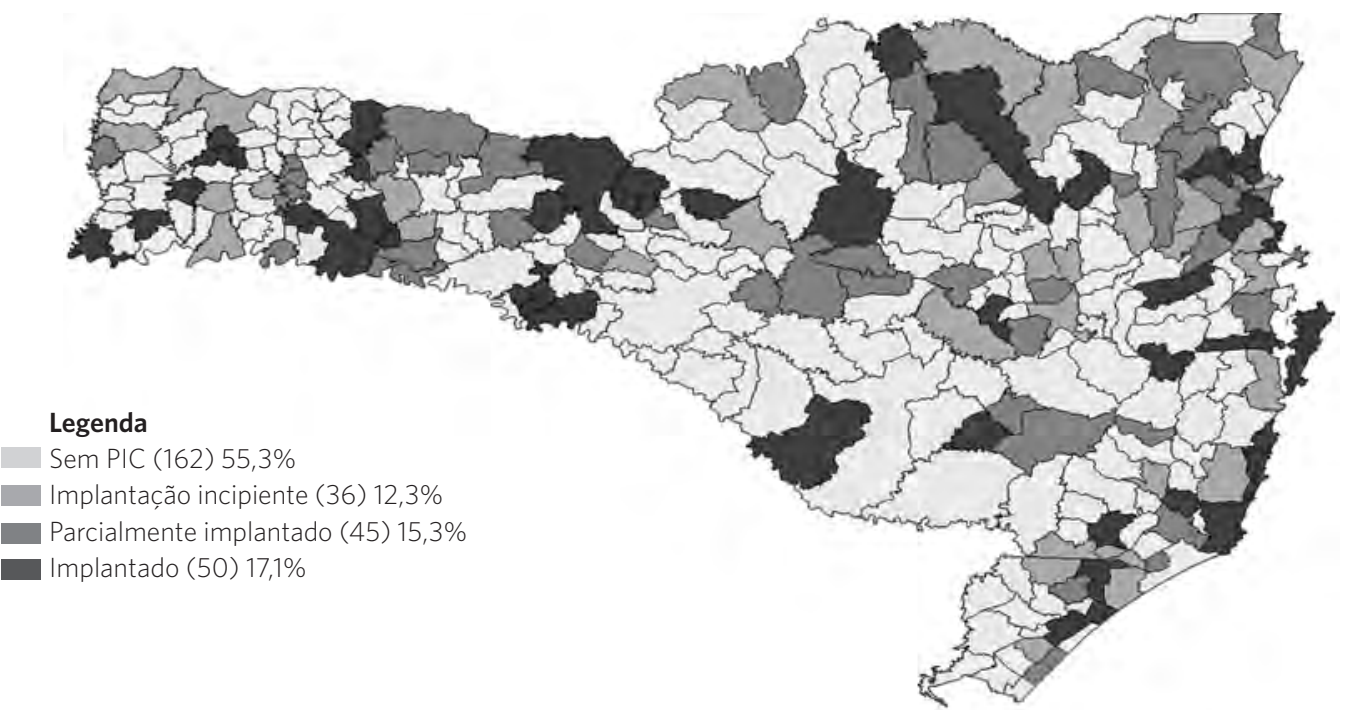

A figura 2 demonstra que a maioria dos municípios de SC (55,3\%) ainda não oferece PIC em seus territórios. Analisando os 131 que apresentaram baixo, médio e alto grau de implantação, 50 (38,2\%) possuíam a maior quantidade de municípios com o juízo de valor ‘implantado’ ou seja, com alto grau de implantação.
Também foi possível identificar no mapa que a implantação das PIC está distribuída pelo estado de forma heterogênea, não apresentando tendências geográficas específicas, apesar de alguns municípios vizinhos apresentarem o contexto de implantação similar, o que pode caracterizar a influência das experiências pela proximidade dos locais. 
Tabela 2. Distribuição dos municípios segundo a classificação geral para a implantação e população potencialmente coberta pelas práticas integrativas e complementares na Atenção Básica em Santa Catarina, Brasil, 2013

\begin{tabular}{|c|c|c|c|c|c|c|c|c|}
\hline \multirow[t]{2}{*}{ Classificação } & \multicolumn{2}{|c|}{ Sem PIC } & \multicolumn{2}{|c|}{$\begin{array}{c}\text { Implantação } \\
\text { incipiente }\end{array}$} & \multicolumn{2}{|c|}{$\begin{array}{l}\text { Parcialmente } \\
\text { implantado }\end{array}$} & \multicolumn{2}{|l|}{ Implantado } \\
\hline & $n$ & $\%$ & $\mathrm{n}$ & $\%$ & $n$ & $\%$ & $n$ & $\%$ \\
\hline \multicolumn{9}{|l|}{ População } \\
\hline$\leq 5$ mil hab $(n=105)$ & 70 & 66,7 & 1 & 1,0 & 14 & 13,3 & 20 & 19,0 \\
\hline $5-20$ mil hab $(n=124)$ & 76 & 61,3 & 15 & 12,1 & 19 & 15,3 & 14 & 11,3 \\
\hline 20 어 50 mil hab $(n=37)$ & 12 & 32,4 & 11 & 29,7 & 6 & 16,2 & 8 & 21,6 \\
\hline$>50$ mil hab $(n=27)$ & 4 & 14,8 & 8 & 29,6 & 7 & 25,9 & 8 & 29,6 \\
\hline Total $(n=293)$ & 162 & 55,3 & 35 & 11,9 & 46 & 15,7 & 50 & 17,1 \\
\hline $\begin{array}{l}\text { População potencial- } \\
\text { mente coberta } \\
(n=6.612 .939)\end{array}$ & 1.649 .998 & 25,0 & 1.238 .285 & 18,7 & 1.760 .519 & 26,6 & 1.964 .137 & 29,7 \\
\hline
\end{tabular}

Fonte: Elaboração própria.

A tabela 2 apresenta os resultados dos graus de implantação das PIC por porte populacional dos municípios. Observa-se que, quando considerados apenas os municípios que ofertam PIC, aqueles com menos de cinco mil habitantes apresentam os melhores resultados, havendo, portanto, alto grau de implantação em 20 dos 35 municípios.

Quanto à análise da classificação geral para implantação e população potencialmente coberta pelas PIC, apresentada também na tabela 2, tem-se que 1.964 .137 (29,7\%) habitantes estão cobertos pelas PIC em seus territórios. E, somando os juízos de valor 'implantado' e 'parcialmente implantado', as PIC cobrem 3.724 .656 (56,3\%) habitantes do estado de Santa Catarina, representando que a maioria da população do estado possui acesso às PIC.

Estudos demonstram que há fatores que podem não ter contribuído para a implantação de experiências em PIC, como a homeopatia, associando motivos do desconhecimento do gestor sobre a PNPIC e racionalidade médica homeopática; a ausência de financiamento específico de uma política nacional, para o gestor municipal implementar e manter os serviços, sendo as iniciativas existentes limitadas e descritas pelo apoio financeiro do gestor local. Apesar destes fatores, destaca-se que o processo de implantação vem ocorrendo independentemente de a gestão do SUS promover a capacitação e contratação de profissionais habilitados em PIC, sendo associado à um fenômeno social que precede e transcende o âmbito das políticas públicas (GALHARDI; BARROS; LEITE-MOR, 2013; ISCHKANIAN; SANTOS; TESSER, 2012; PELICIONI, 2012; SOUSA ET AL., 2012).

Para que o gestor municipal viabilize a implantação de ações e serviços em PIC, é necessário um planejamento administrativo, político e institucional junto aos profissionais e sociedade civil, sustentado na gestão participativa, na apropriação democrática dos instrumentos de gestão e atenção às especificidades loco regionais. Ainda é necessário reconhecer que esse processo de implantação prevê algumas fases: feitura de pactos do núcleo responsável; mapeamento dos profissionais capacitados existentes; regulamentação e legitimação por meio de trâmites e normas legais; desenvolvimento de atividades de tutorias e atividades de educação permanente (BARRETO; NUNES; AROUCHA, 2014; SANTOS; TESSER, 2012;). Importante destacar que para a efetiva implantação, é necessário superar os ciclos 
da mudança dos governantes, o que impede a continuidade das ações em PIC. Para isso, é necessário promover uma mudança cultural, a fim de legitimar socialmente as PIC.

Sob o eixo fundamental da lógica de pensamentos que deram origem a este modelo de atenção em que as PIC estão inseridas, parte-se de algumas reflexões e constatações. Por exemplo, as PIC permitem um olhar diferenciado sobre o indivíduo e o seu processo saúde-doença, não somente como uma prática de cuidado, mas como uma estratégia que promove mudanças no modelo assistencial hegemônico em saúde, que, muitas vezes, exclui outras formas de produzir e legitimar saberes e práticas. Também, a acentuação da autonomia do usuário promove autocuidado; a afirmação de um saber que tem como categoria central a saúde e não a doença, traduz o cuidado não ao ato da assistência às doenças, mas para a atenção à promoção da saúde das pessoas e coletividades; o potencial desmedicalizante da vida, desapropria o processo de normalização impostos pelo modelo biomédico e constante medicalização pelas indústrias farmacêuticas, trazendo uma visão integrada da pessoa, e não de suas partes (BARRETO; NUNES; AROUCHA, 2014; OTANI; BARROS, 2011; SANTOS; TESSER, 2012;).

\section{Considerações finais}

A análise realizada permitiu avaliar os municípios pesquisados do estado de Santa Catarina quanto ao grau de implantação das práticas integrativas e complementares na AB. Apresentou resultado de implantação expressivo, com diferenças entre os municípios e seus portes populacionais, visto que a maioria da população do estado possui acesso às PIC; refletindo 56,3\% do estado com PIC implantadas ou parcialmente implantadas.

Considera-se que o modelo proposto tenha demonstrado viabilidade de aplicação para municípios de diferentes portes populacionais e que o conjunto de indicadores foi considerado adequado para a avaliação do grau de implantação das práticas integrativas e complementares na $\mathrm{AB}$ por meio de dados secundários. Os dados coletados permitiram gerar as informações necessárias para a avaliação, mas foram considerados limitantes pela impossibilidade de elaboração de alguns indicadores que avaliassem outras características consideradas importantes para implantação, tais como características de profissionais e tipos de equipe, outros insumos para PIC, financiamento, dentre outros.

Quanto à análise do alcance das metas propostas pelas recomendações para implantação pelas instâncias superiores (MS e DAB), identificaram-se insuficiências, dado que os códigos de classificação de PIC definidos pela Portaria $n^{0} 853 / 2006$ são inadequados para representar a realidade dos municípios, que apresentam a oferta de outras PIC não estabelecidas na PNPIC, demonstrando uma subnotificação da oferta desse serviço.

Mas também identificaram-se avanços via diretrizes da PNPIC. A implantação das PIC no território catarinense está avançada, pois os indicadores da dimensão Assistência, de maneira geral, foram responsáveis pela grande quantidade de juízos de valor 'bom', refletindo que as EABs estão em busca de capacitação na área e ofertam as PIC para a população em seus territórios, auxiliando o acesso dos usuários do SUS a esse novo modelo de atenção à saúde.

Deve-se ressaltar, também, a provável interferência dos contextos locais na implantação das práticas integrativas e complementares, o que, neste estudo, não foi possível identificar. Sugerem-se pesquisas futuras para aprofundamento dessas questões, de modo a que possam responder às inquietações reveladas pelos dados.

Considera-se que, para ampliar a implantação das PIC na $\mathrm{AB}$, a gestão federal deva assumir de fato a PNPIC como uma política de Estado, garantindo recursos financeiros para sua atuação no SUS e permitindo que o esforço das equipes que ofertam PIC 
transformem-se em experiências mais exitosas, não permanecendo apenas uma política focal e isolada.

$A$ avaliação da $A B$ no âmbito do PMAQ-AB possibilita reconhecer as características das EABs e suas ações nesse nível de atenção. Ainda que possa ser considerado um programa novo e em fase de expansão, ele deve ser assumido e institucionalizado por alguns potenciais: reconhecimento de que as PIC fazem parte de um componente importante de qualidade para a $\mathrm{AB}$ por meio do monitoramento das equipes que tenham iniciativas em PIC nos diversos contextos do Brasil, contribuindo para a pluralidade de escolhas terapêuticas de cuidados em saúde; levantamento do importante lugar da institucionalização da avaliação na $\mathrm{AB}$, não como um componente que penaliza mas como estratégia de indução da melhoria de indicadores assistenciais, estruturais e gerenciais em todo território nacional; e sua assunção como um importante instrumento de coleta de dados, com potencial para subsidiar a tomada de decisão da gestão para a garantia da atenção integral e universal aos usuários do SUS.

É preciso também levantar algumas deficiências do programa: as PIC são avaliadas no PMAQ-AB, mas as equipes não são remuneradas por realizá-las; as informações derivadas do banco de dados correspondem ao conjunto de $\mathrm{EAB}$ que aderiu voluntariamente ao programa, pressupondo um viés de seleção com tendência de acentuação da positividade das respostas das equipes, por se tratar de uma coleta de dados que alia o resultado do desempenho das equipes para fins de transferências financeiras; e o repasse não prevê um sistema de recompensas que atinja diretamente o trabalhador das EABs.

Outra possível limitação do estudo diz respeito ao fato de as variáveis obtidas do PMAQ-AB não permitirem estabelecer relações entre processo e estrutura das PIC identificadas, embora sejam suficientes para se avaliar o grau de implantação dessas práticas.

Em conclusão, os resultados desta pesquisa sugerem diversos caminhos para o aprimoramento das PIC. Estudos futuros poderão avaliar o grau de implantação das práticas integrativas e complementares em diferentes estados brasileiros, bem como aprimorar o modelo avaliativo a partir de propostas mais recentes de ciclos posteriores do PMAQ-AB. Este registro pode ser o início de um processo consistente de avaliação e de contínuo aperfeiçoamento da forma de organização das práticas integrativas e complementares no âmbito do sistema de saúde brasileiro.

\section{Colaboradores}

LN Losso foi responsável pela coleta de dados e realização da revisão de literatura. LN Losso e SFT Freitas delinearam o estudo, analisaram os dados, escreveram o artigo e fizeram a revisão final do artigo.

\section{Agradecimentos}

Ao MS e DAB, pelos dados desta pesquisa. Ao professor Fúlvio Nedel, pelo auxílio na coleta do banco de dados e análises iniciais. 


\section{Referências}

BAHIA, L. O sistema de saúde brasileiro entre normas e fatos: universalização mitigada e estratificação subsidiada. Ciência \&t Saúde Coletiva, Rio de Janeiro , v. 14, n. 3, p. 753-762, jun. 2009. Disponível em: <http://www. scielo.br/scielo.php?script=sci_arttext\&pid=S1413$-81232009000300011 \& \operatorname{lng}=\mathrm{en} \& \mathrm{nrm}=\mathrm{iso}>$. Acesso em: 7 nov. 2016

BARRETO, J. A.; NUNES, J. G.; AROUCHA, E. B. L. Um olhar trimembrado sobre a implantação de serviços de práticas integrativas e complementares em saúde. In: Práticas integrativas em saúde: proposições teóricas e experiências na saúde e educação. Recife: UFPE, 2014. p. 155-174. Disponível em: <http://www.neplame. univasf.edu.br/uploads/7/8/9/0/7890742/livro_praticas_integrativas_em_sa\%C3\%BAde_versao_final.pdf > Acesso em: 10 abr. 2017.

BRASIL. Ministério da Saúde. População residente. Estimativas para o TCU Santa Catarina. Brasília, DF, 2013a. Disponível em: <http://tabnet.datasus.gov.br/ cgi/tabcgi.exe?ibge/cnv/poptsc.def $>$. Acesso em: 17 nov. 2015

Ministério da Saúde. Portaria $n^{\circ}$ 971, de 3 de maio de 2006. Aprova a Política Nacional de Práticas Integrativas e Complementares (PNPIC) no Sistema Único de Saúde. Diário Oficial [da] República Federativa do Brasil, Brasília, DF, 3 maio 2006a. Disponível em: <http://bvsms.saude.gov.br/bvs/saudelegis/gm/2006/ prt0971_03_05_2006.html>. Acesso em: 10 maio 2015.

Ministério da Saúde. Portaria n ${ }^{\circ} 853$, de 17 de novembro de 2006. Inclui na Tabela de Serviços/ classificações do Sistema de Cadastro Nacional de Estabelecimentos de Saúde - SCNES de Informações do SUS, o serviço de código 068 - Práticas Integrativas e Complementares. Diário Oficial [da] República Federativa do Brasil, Brasília, DF, 18 nov. 2006b. Disponível em: <http://189.28.128.100/dab/docs/legislacao/portaria853_17_11_06. Pdf>. Acesso em: 10 set. 2014.

Ministério da Saúde. Portaria n ${ }^{\circ} 1.654$, de 19 de julho de 2011. Institui, no âmbito do Sistema Único de
Saúde, o Programa Nacional de Melhoria do Acesso e da Qualidade da Atenção Básica (PMAQ-AB) e o Incentivo Financeiro do PMAQ-AB, denominado Componente de Qualidade do Piso de Atenção Básica Variável - PAB Variável. Diário Oficial [da] República Federativa do Brasil, Brasília, DF, 2011.

Ministério da Saúde. Portaria nº 533 , de 28 de março de 2012. Estabelece o elenco de medicamentos e insumos da Relação Nacional de Medicamentos (RENAME) no âmbito do Sistema Único de Saúde (SUS). Diário Oficial [da] República Federativa do Brasil, Brasília, DF, 3 maio 2012a. Disponível em: <http://bvsms.saude.gov.br/bvs/saudelegis/gm/2012/ prt0533_28_03_2012.html>. Acesso em: 10 ago. 2015.

Ministério da Saúde. Teto, credenciamento e implantação das estratégias de Agentes Comunitários de Saúde, Saúde da Família e Saúde Bucal. Brasília, DF, $2013 b$.

Ministério da Saúde. Secretaria de Atenção à Saúde. Instrumento de Avaliação Externa para as Equipes de Atenção Básica: Saúde da Família e Equipe Parametrizada. Brasília, DF, 2013c.

Ministério da Saúde. Secretaria de Atenção à Saúde. Departamento de Atenção Básica. Política Nacional de Atenção Básica. Brasília, DF, 2012 b. Disponível em: <http://189.28.128.100/dab/docs/publicacoes/geral/pnab.pdf>. Acesso em: 10 set. 2014.

- Ministério da Saúde. Secretaria de Atenção à Saúde. Departamento de Atenção Básica. Práticas integrativas e complementares: plantas medicinais e fitoterapia na Atenção Básica/Ministério da Saúde. Brasília, DF, 2012c.

CAVALCANTI, F. et al. Política Nacional de Práticas Integrativas e Complementares no SUS: histórico, avanços, desafios e perspectivas. In: BARRETO, A. F. (Org.). Práticas integrativas em saúde: proposições teóricas e experiências na saúde e educação. Recife: UFPE, 2014. p. 139-153. Disponível em: <http://www. neplame.univasf.edu.br/uploads/7/8/9/0/7890742/ 
livro_praticas_integrativas_em_sa\%C3\%BAde_versao_final.pdf>. Acesso em: 10 abr. 2017.

CONTANDRIOPOULOS, A. P. Reformar o sistema de saúde: uma utopia para sair de um status quo inaceitável. Saúde em Debate, Rio de Janeiro, n. 49-50, dez. 1995/mar. 1996. p. 53-64. Disponível em: <http://docvirt.com/asp/acervo_cebes.asp?Bib=SAUDEDEBAT E\&PASTA $=$ N.49\&pesq $=$ contandriopoulos $\& \mathrm{x}=0 \& \mathrm{y}=0>$. Acesso em: 18 out. 2016.

FAUSTO, M. C. R., et al. A posição da Estratégia Saúde da Família na rede de atenção à saúde na perspectiva das equipes e usuários participantes do PMAQ-AB. Saúde em Debate, Rio de Janeiro, v. 38, n. esp., p. 13-33, out. 2014. Disponível em: <http://www. scielo.br/scielo.php?script=sci_arttext\&pid=S0103$-11042014000600013 \& \operatorname{lng}=\mathrm{en} \& \mathrm{nrm}=\mathrm{iso}>$. Acesso em: 10 abr. 2017.

FONSECA SOBRINHO, D. et al. Compreendendo o apoio matricial e o resultado da certificação de qualidade nas áreas de atenção à criança, mulher, diabetes/ hipertensão e saúde mental. Saúde em Debate, Rio de Janeiro, v. 38, n. esp., p. 83-93, out. 2014. Disponível em: <http://www.scielo.br/scielo.php?script=sci arttext\&pid=S0103-11042014000600083\&lng=en\&nrm =iso $>$. Acesso em: 10 abr. 2017.

GALHARDI, W. M. P.; BARROS, N. F.; LEITE-MOR, A. C. M. B. O conhecimento de gestores municipais de saúde sobre a Política Nacional de Prática Integrativa e Complementar e sua influência para a oferta de homeopatia no Sistema Único de Saúde local. Ciência \&t Saúde Coletiva, Rio de Janeiro, v. 18, n. 1, p. 213-220, jan. 2013 . Disponível em: <http://www. scielosp.org/scielo.php?script=sci_arttext\&pid=S1413$-81232013000100022 \& \operatorname{lng}=$ pt\&nrm $=$ iso $>$. Acesso em: 10 abr. 2017.

HARTZ, Z. M. A. et al. Avaliação do Programa Materno Infantil: análise de implantação em sistemas locais de saúde no nordeste do Brasil. In: HARTZ, Z. M. A. (Org.). Avaliação em saúde: dos modelos conceituais à prática na análise da implantação de programas. Rio de Janeiro: Fiocruz, 1997. p. 89-131. Disponível em: <http://
www.faa.br/PDF/livros_eletronicos/medicina/6_ Avaliacao_em_saude.pdf >. Acesso em: 13 jul. 2015.

ISCHKANIAN, P. C.; PELICIONI, M. C. F. Desafios das práticas integrativas e complementares no SUS visando a promoção da saúde. Revista Brasileira de Crescimento e Desenvolvimento Humano, São Paulo, v. 22, n. 2, p. 233-238, 2012. Disponível em: <http://pepsic.bvsalud. org/pdf/rbcdh/v22n2/pt_16.pdf >. Acesso em: 10 abr. 2017.

MEDINA, M. G., et al. Uso de modelos teóricos na avaliação em saúde: aspectos conceituais e operacionais. In: HARTZ, Z. M. A.; SILVA, L. M. V. (Org.). Avaliação em saúde: dos modelos teóricos à prática na avaliação de programas e sistemas de saúde. Salvador: EDUFBA; Rio de Janeiro: Fiocruz, 2005, p. 41-64.

NASCIMENTO, M. C. et al. A categoria racionalidade médica e uma nova epistemologia em saúde. Ciência \& Saúde Coletiva, Rio de Janeiro, v. 18, n. 12, p. 3595-3604, dez. 2013. Disponível em: <http://www. scielo.br/scielo.php?script=sci_arttext\&pid=S1413$-81232013001200016 \& \operatorname{lng}=\mathrm{en} \& \mathrm{nrm}=\mathrm{iso}>$. Acesso em: 10 out. 2017.

OTANI, M. A. P.; BARROS, N. F. A Medicina Integrativa e a construção de um novo modelo na saúde. Ciência Et Saúde Coletiva, Rio de Janeiro, v. 16, n. 3, p. 1801-1811, mar. 2011. Disponível em: <http://www. scielo.br/scielo.php?script=sci_arttext $\&$ pid $=$ S1413$-81232011000300016 \& \operatorname{lng}=\mathrm{en} \& \mathrm{nrm}=\mathrm{iso}>$. Acesso em: 10 abr. 2017.

SANTOS, M. C.; TESSER, C. D. Um método para a implantação e promoção de acesso às Práticas Integrativas e Complementares na Atenção Primária à Saúde. Ciência \& Saúde Coletiva, Rio de Janeiro, v. 17, n. 11, p. 3011-3024, nov. 2012. Disponível em: <http://www.scielo.br/scielo.php?script=sci arttext\&pid=S1413-81232012001100018\&lng=en\&nrm=i so>. Acesso em: 10 abr. 2017.

SEIDL, H. et al. Gestão do trabalho na Atenção Básica em Saúde: uma análise a partir da perspectiva das equipes participantes do PMAQ-AB. 
Saúde em Debate, Rio de Janeiro, v. 38, n. esp., p. 94-108, out. 2014. Disponível em: <http://www. scielo.br/scielo.php?script=sci_arttext\&pid=S0103$-11042014000600094 \& \operatorname{lng}=$ en\&nrm=iso $>$. Acesso em: 10 abr. 2017.

SILVA, L. M. V.; FORMIGLI, V. L. A. Avaliação em Saúde: Limites e Perspectivas. Caderno de Saúde Pública, Rio de Janeiro, v. 10, n. 1, p. 80-91, jan./mar., 1994.

SOUSA, I. M. C. et al. Práticas integrativas e complementares: oferta e produção de atendimentos no SUS e em municípios selecionados. Caderno de Saúde Pública, Rio de Janeiro, v. 28, n. 11, p. 2143-2154, nov.
2012 . Disponível em: <http://www.scielo.br/scielo. php?script=sci_arttext\&pid=S0102-311X201200110001 $4 \& \operatorname{lng}=\mathrm{en} \& \mathrm{nrm}=\mathrm{iso}>$. Acesso em: 5 fev. 2017.

WORLD HEALTH ORGANIZATION (WHO).

Estrategia de la OMS sobre medicina tradicional 20142023. Genebra, 2013. Disponível em: <http://apps.who. int/medicinedocs/documents/s21201es/s2120les.pdf $>$. Acesso em: 5 abr. 2017.

Recebido para publicação em abril de 2017

Versão final em agosto de 2017

Conflito de interesses: inexistente

Suporte financeiro: não houve 